\title{
A Dual-Band Fabry-Pérot Cavity Antenna with a Single Partially Reflecting Surface and Reduced Cavity Height for WLAN Applications
}

\author{
Sayan SARKAR, Bhaskar GUPTA \\ Dept. of ETCE, Jadavpur University, 188 Raja S.C. Mallick Rd, Kolkata - 700032, India \\ sayansarkar0794@gmail.com, gupta_bh@yahoo.com
}

Submitted October 9, 2020 / Accepted January 27, 2021

\begin{abstract}
This paper proposes a dual-band Fabry-Pérot Cavity Antenna (FPCA) operating at two important WLAN bands - the $2.4 \mathrm{GHz}$ band and $5.8 \mathrm{GHz}$ band. It exhibits Circular Polarization (CP) at $2.4 \mathrm{GHz}$ and Linear Polarization (LP) at $5.8 \mathrm{GHz}$. The proposed antenna uses only a single Partially Reflecting Surface (PRS) layer to achieve good $3 d B$ gain bandwidths in both the bands. The cavity height of the antenna is also significantly reduced by using an Artificial Magnetic Conductor (AMC) ground plane. The antenna achieves a $3 d B$ axial ratio bandwidth $(A R-B W)$ of $7.9 \%$ at $2.4 \mathrm{GHz}$ with peak measured gains of $14.3 \mathrm{dBi}$ and $15.5 \mathrm{dBi}$ at $2.4 \mathrm{GHz}$ and $5.8 \mathrm{GHz}$ respectively. The antenna also exhibits a $3 \mathrm{~dB}$ gain $B W$ of $7.8 \%$ in the first band and $5.5 \%$ in the second band.
\end{abstract}

\section{Keywords}

Artificial Magnetic Conductor (AMC), Circular Polarization (CP), dual-band, Fabry-Pérot Cavity Antenna (FPCA), Partially Reflecting Surface (PRS), positive reflection phase gradient, WLAN

\section{Introduction}

Compact metamaterial based antennas have become very popular in recent times [1-3]. Owing to their small size, they have small values of gain. They can be used in applications where the antenna size is a constraint. However, in some applications, the antenna size is not a factor and very high gain antennas are required. For such applications, Fabry-Pérot Cavity antennas are the best candidates.

In 1956, Giswalt von Trentini demonstrated that by placing a partially reflecting sheet in front of an antenna with a reflecting screen, the directivity of the antenna is greatly enhanced [4]. Such cavities, consisting of a fully reflecting bottom wall and a partially reflecting top wall, are called Fabry-Pérot Cavities (FPCs). When an antenna is placed inside the cavity near the bottom wall, the resulting system is referred to as a Fabry-Pérot Cavity Antenna (FPCA). The resonance condition of the FPC is given by the following equation:

$$
h=\frac{c}{4 \pi f_{\mathrm{r}}}\left(\varphi_{\mathrm{PRS}}+\varphi_{\mathrm{GND}}-2 \pi k\right), \quad k=0,1,2, \ldots
$$

where $c$ is speed of light in vacuum, $h$ is cavity height, $f_{\mathrm{r}}$ is cavity resonant frequency, $\varphi_{\mathrm{PRS}}$ and $\varphi_{\mathrm{GND}}$ are reflection coefficient phases of the PRS and ground plane, respectively. The radiation that leaks through the PRS at resonance is highly directive due to constructive interference of the fields inside the cavity. This directivity enhancement property of the FPC has been exploited to design many high gain antennas [5-9].

One inherent disadvantage of conventional FPCAs is their narrow $3 \mathrm{~dB}$ gain bandwidths. This is because the cavity resonance condition is satisfied by only a single frequency, $f_{\mathrm{r}}$ and the field interference within the cavity becomes less constructive as we move away from $f_{\mathrm{r}}$. This leads to a reduction in the directivity of the radiated beam. The $3 \mathrm{~dB}$ gain bandwidth $(\mathrm{BW})$ is therefore an effective measure of how wideband an FPCA actually is.

One way to increase the $3 \mathrm{~dB}$ gain BW of FPCAs is by using a PRS whose reflection coefficient phase increases with frequency. This ensures that the resonance condition of (1) is satisfied even when the frequency is increased. This method is used in [10] and [11] to achieve wider $3 \mathrm{~dB}$ gain BWs. However, both these antennas use two or more PRS layers to achieve a wider bandwidth. This leads to an increased cavity height and bulky configuration. The positive reflection phase gradient can also be achieved by using a single PRS layer. In [12], the authors use a single PRS layer with metallization on both sides of the substrate to achieve a positive reflection phase gradient.

The FPCA proposed in this paper operates in two important wireless communication bands - the $2.4 \mathrm{GHz}$ band and $5.8 \mathrm{GHz}$ band. They are popularly referred to as the WLAN bands. The proposed antenna radiates a Right Hand $\mathrm{CP}$ (RHCP) wave at $2.4 \mathrm{GHz}$ and a linearly polarized (LP) wave at $5.8 \mathrm{GHz}$. The antenna consists of only a single PRS layer and exhibits a positive reflection phase gradient in both the $2.4 \mathrm{GHz}$ and $5.8 \mathrm{GHz}$ bands. An Artificial Magnetic Conductor (AMC) is used as the antenna ground plane. The cavity height of the FPCA is reduced significantly by manipulating the reflection phases of the PRS 
and the AMC ground plane. The dimensions of the proposed antenna are $1.5 \lambda_{2.4 \mathrm{GHz}} \times 1.5 \lambda_{2.4 \mathrm{GHz}} \times 0.18 \lambda_{2.4 \mathrm{GHz}}$ $(187.5 \mathrm{~mm} \times 187.5 \mathrm{~mm} \times 22.5 \mathrm{~mm})$ where $\lambda_{2.4 \mathrm{GHz}}$ is free space wavelength at $2.4 \mathrm{GHz}$. The FPC significantly enhances the gain of the antenna in both the frequency bands.

The proposed antenna has a greatly reduced cavity height and only a single PRS layer which makes it compact and rigid. It also has high gains and wide $3 \mathrm{~dB}$ gain $\mathrm{BWs}$ in both bands. Circular polarization at $2.4 \mathrm{GHz}$ further enhances communication by mitigating orientation mismatch loss between transmitter and receiver.

\section{Design of PRS and AMC Unit Cells}

All the simulations of this paper have been performed using CST Studio Suite. The analysis of the unit cells have been performed with periodic boundary conditions along both axes ( $x$ axis and $y$ axis) in the plane of the unit cell. Both the antenna with AMC ground plane and the PRS are designed on FR-4 substrates $\left(\varepsilon_{\mathrm{r}}=4.2, \tan \delta=0.02\right.$, thickness $=1.52 \mathrm{~mm}$ ).

\subsection{PRS Unit Cells}

In [12], the authors use two periodic arrays of closely resonating structures, each printed on the opposite side of a dielectric substrate, to design a PRS which achieves a positive reflection phase gradient.

Using the same principle, the proposed antenna also consists of two arrays of closely resonating elements printed on either side of the FR-4 substrate. However, the resonating elements used in this paper are different from the ones used in [12]. The top and bottom unit cells of the proposed PRS are shown in Fig. 1.

The top unit cell consists of a cross-shaped printed metallic structure while the bottom unit cell consists of a square patch present inside a square loop. The resonating structures on the top (Fig. 1(a)) and bottom (Fig. 1(b)) together help to create a positive reflection phase gradient. The dimensions of the top and bottom unit cells are optimized by varying the gaps and element dimensions until the positive reflection phase gradients lie within the two bands of operation.

The simulated phases and magnitudes of the reflection coefficients in the two bands are shown in Fig. 2. The pos-

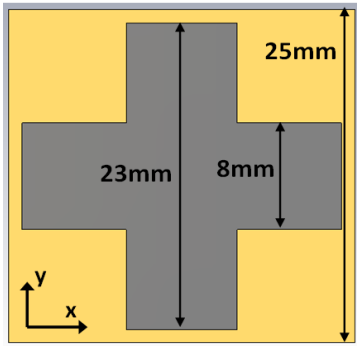

(a)

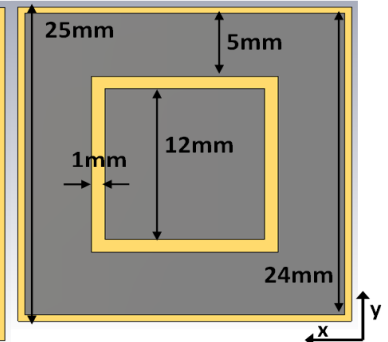

(b)
Fig. 1. PRS unit cell: (a) top and (b) bottom.

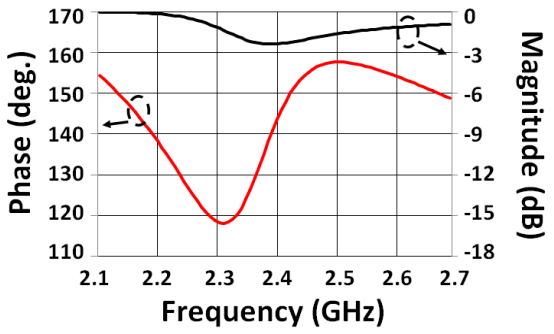

(a)

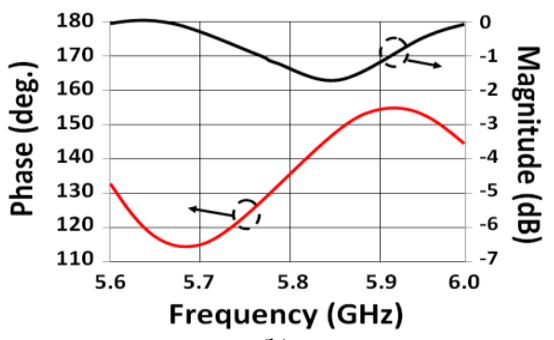

(b)

Fig. 2. Reflection coefficient magnitudes and phases of the PRS in the (a) $2.4 \mathrm{GHz}$ band and (b) $5.8 \mathrm{GHz}$ band.

itive reflection phase gradient extends from around $2.3 \mathrm{GHz}$ to $2.5 \mathrm{GHz}$ in the first band and from $5.7 \mathrm{GHz}$ to $5.9 \mathrm{GHz}$ in the second band. It can be seen that the reflection coefficient magnitudes are quite high, which should result in high gain enhancement [4].

The reflection phases at $2.4 \mathrm{GHz}$ and $5.8 \mathrm{GHz}$ are $145^{\circ}$ and $135^{\circ}$ respectively. These values will be needed in calculations performed in the following section.

The E-field distributions on the top and bottom unit cells of the PRS are shown in Fig. 3. In the bottom unit cell, the majority of the E-fields are concentrated in the gap between the outer square loop and inner square patch at both $2.4 \mathrm{GHz}$ and $5.8 \mathrm{GHz}$. In the top unit cell, the E-fields are concentrated along the upper and lower portions of the cross at $2.4 \mathrm{GHz}$ and along the four corners of the cross at $5.8 \mathrm{GHz}$. The interactions of the top and bottom unit cells help in generating positive reflection phase gradients at both $2.4 \mathrm{GHz}$ and $5.8 \mathrm{GHz}$.

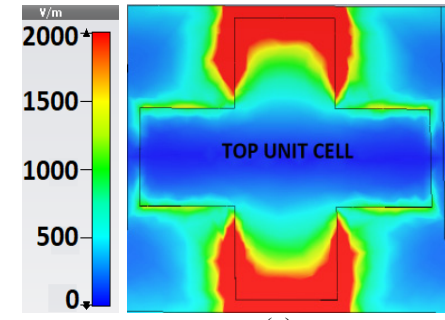

(a)

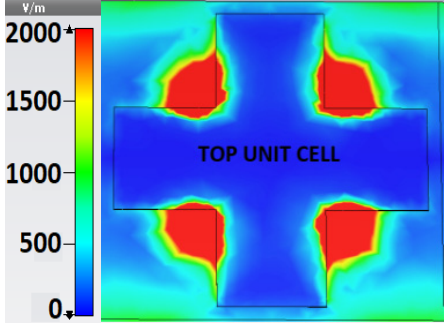

(c)

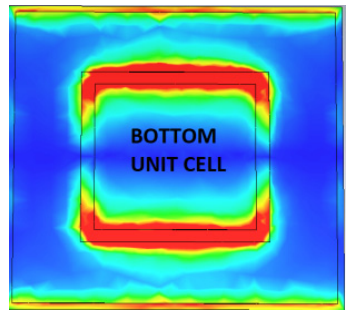

(b)

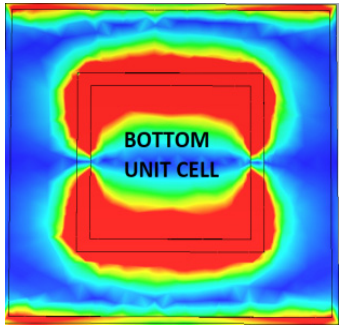

(d)
Fig. 3. E-field distributions (V/m) at (a, b) $2.4 \mathrm{GHz}$ and (c, d) $5.8 \mathrm{GHz}$. 


\subsection{AMC Ground Plane}

Equation (1) tells us that the Fabry-Perot cavity height $h$ depends on the reflection phases of both the PRS layer and the ground plane (it depends on the sum of $\varphi_{\mathrm{PRS}}$ and $\left.\varphi_{\mathrm{GND}}\right)$. Therefore, $h$ can be reduced significantly by manipulating $\varphi_{\mathrm{GND}}$ and $\varphi_{\mathrm{PRS}}$. Variations of $\varphi_{\mathrm{GND}}$ can be achieved by using an AMC ground plane instead of the normal metallic ground plane which has a fixed $\varphi_{\mathrm{GND}}$ of $\pi$ radians.

If the cavity height $h$, resonance frequency $f_{\mathrm{r}}$ and PRS reflection phase $\varphi_{\mathrm{PRS}}$ are fixed, then the value of $\varphi_{\mathrm{GND}}$ required to satisfy equation (1) can be calculated. In this paper, $h$ is fixed at $\lambda_{2.4 \mathrm{GHz}} / 6(\approx 20 \mathrm{~mm})$. The reflection coefficient phase of the PRS, $\varphi_{\mathrm{PRS}}$, is $145^{\circ}$ at $2.4 \mathrm{GHz}$ and $135^{\circ}$ at $5.8 \mathrm{GHz}$ (see Sec. 2.1).

To calculate the required value of $\varphi_{\mathrm{GND}}$ at $2.4 \mathrm{GHz}$, we put $f_{\mathrm{r}}=2.4 \mathrm{GHz}, \varphi_{\mathrm{PRS}}=145^{\circ}, k=0$ and $h=20 \mathrm{~mm}$. This yields $\varphi_{\mathrm{GND}}=-25^{\circ}$. The required value of $\varphi_{\mathrm{GND}}$ at $5.8 \mathrm{GHz}$ can be calculated similarly by putting $f_{\mathrm{r}}=5.8 \mathrm{GHz}, \varphi_{\mathrm{PRS}}=$ $135^{\circ}, k=1$ and $h=20 \mathrm{~mm}$. This results in $\varphi_{\mathrm{GND}}=144^{\circ}$.

Therefore, the designed AMC ground plane should exhibit a reflection phase of $-25^{\circ}$ at $2.4 \mathrm{GHz}$ and $144^{\circ}$ at $5.8 \mathrm{GHz}$ in order to satisfy the cavity resonance condition. The designed unit cell of the AMC ground plane and its reflection coefficients are shown in Fig. 4. It can be seen that the reflection phases are very close to the calculated values at both $2.4 \mathrm{GHz}$ and $5.8 \mathrm{GHz}$.

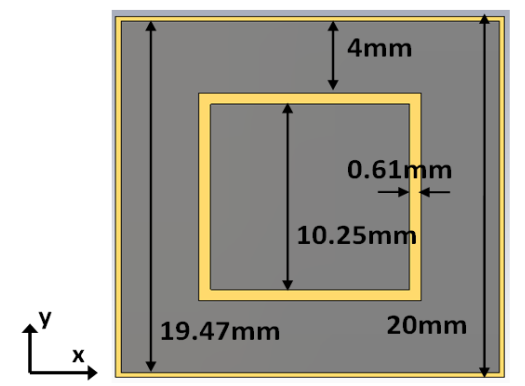

(a)

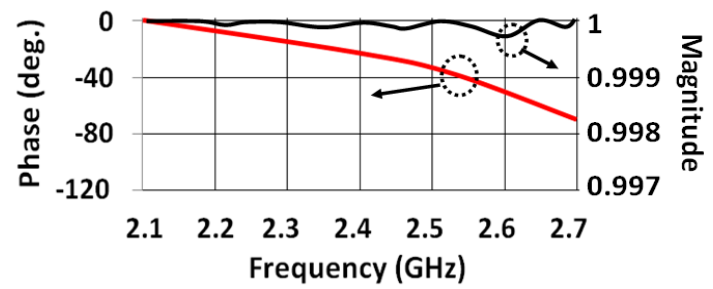

(b)

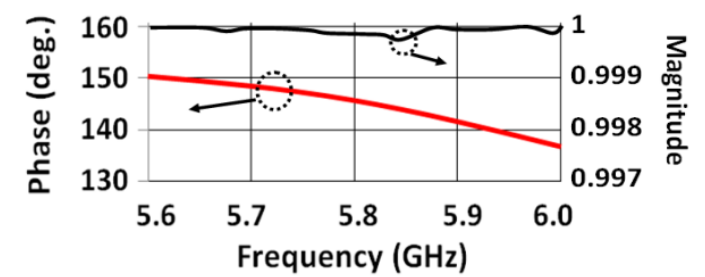

(c)

Fig. 4. (a) Designed AMC unit cell and its reflection coefficients in the (b) $2.4 \mathrm{GHz}$ band and (c) $5.8 \mathrm{GHz}$ band.
Observing the reflection phases of the PRS (Fig. 2(a), (b)) and AMC (Fig. 4(b), (c)) in the two frequency bands, it can be concluded that $\varphi_{\mathrm{GND}}+\varphi_{\mathrm{PRS}}$ increases with frequency from $2.3 \mathrm{GHz}$ to $2.5 \mathrm{GHz}$ and from $5.7 \mathrm{GHz}$ to $5.9 \mathrm{GHz}$.

\section{Patch Antenna}

A patch antenna with asymmetric cross slots and diagonally opposite truncated corners is designed to act as the radiator. The antenna is fed via a coaxial cable located near the edge of a microstrip line, as shown in Fig. 5.

If a patch antenna is designed with only the corner truncations, it radiates a $\mathrm{CP}$ wave near $2.5 \mathrm{GHz}$. After the addition of the asymmetric cross slots, the patch radiates a CP wave at $2.4 \mathrm{GHz}$ as well as an additional LP wave at $5.8 \mathrm{GHz}$. The current distributions of the patch antenna at 2.4 GHz and 5.8 GHz are shown in Fig. 6.

The addition of the slots therefore serves two purposes. It shifts the first band slightly to the left (from $2.5 \mathrm{GHz}$ to $2.4 \mathrm{GHz}$; due to increased current path length) and adds a second resonance near $5.8 \mathrm{GHz}$.

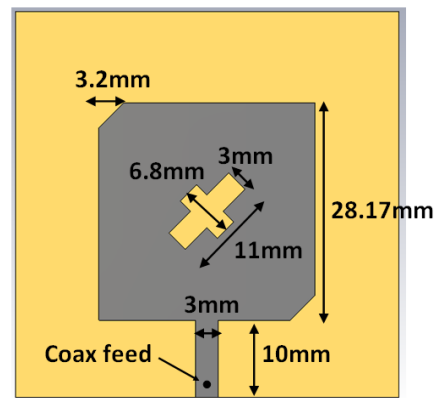

Fig. 5. Designed patch antenna.

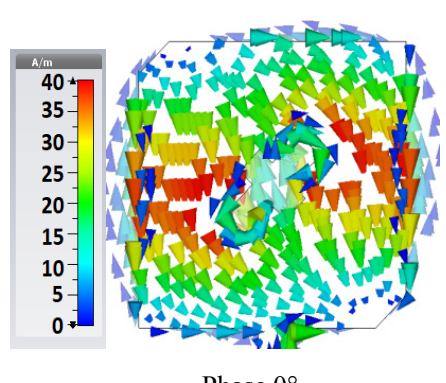

Phase $0^{\circ}$

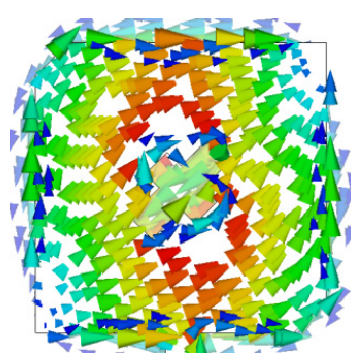

Phase $90^{\circ}$ (a)

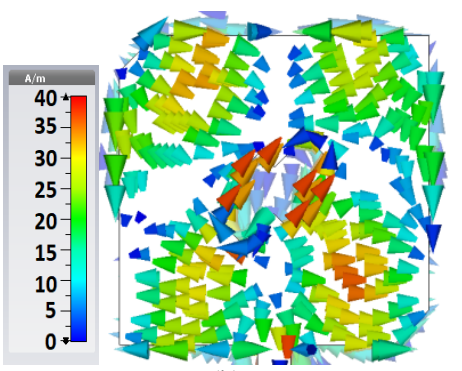

(b)

Fig. 6. Surface current distributions $(\mathrm{A} / \mathrm{m})$ at (a) $2.4 \mathrm{GHz}$ and (b) $5.8 \mathrm{GHz}$ 
The widths of the slots are made equal. Increasing/ decreasing the widths results in left/right shift of the first resonance due to the increased/decreased current path length. The current concentration around the slots at $5.8 \mathrm{GHz}$ can be observed from Fig. 6(b). As a result, the lengths of both the shorter and longer slots affect the $5.8 \mathrm{GHz}$ band. Additionally, the longer slot also affects the $2.4 \mathrm{GHz}$ band. Therefore, the two bands can be adjusted and shifted by varying the lengths of the cross slots.

\section{Fabry-Pérot Cavity Antenna}

The proposed FPCA is shown in Fig. 7. The patch antenna described in the previous section is used as the primary radiator. It is placed on the AMC ground plane where it is surrounded by 40 AMC unit cells. The gaps between the patch and the AMC unit cells are shown in Fig. 7(a). The PRS layer, consisting of 25 unit cells, is placed above the patch antenna-AMC ground plane layer. Although the initial height of the cavity was fixed at $20 \mathrm{~mm}\left(\approx \lambda_{2.4 \mathrm{GHz}} / 6\right)$, the final cavity height was chosen to be $22.5 \mathrm{~mm}$ after optimizations.

\subsection{Measurement Setup}

A vector network analyzer (VNA) from Agilent Technologies having model number E5071B $(300 \mathrm{kHz}$ to $8.5 \mathrm{GHz}$ ) is used for all measurements. The $\mathrm{S}_{11}$ of the antenna under test (AUT) is measured by connecting it to the VNA.

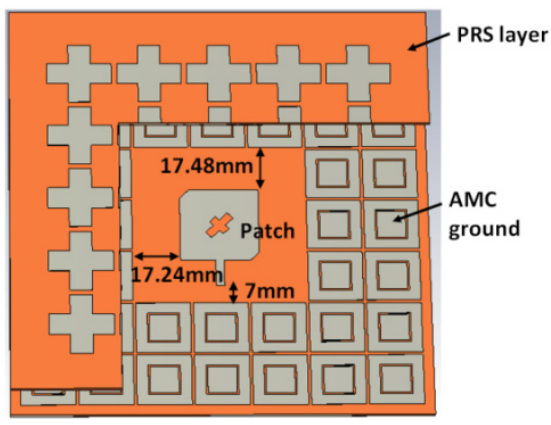

(a)

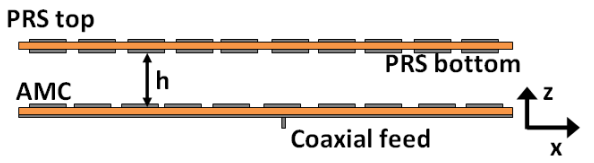

(b)

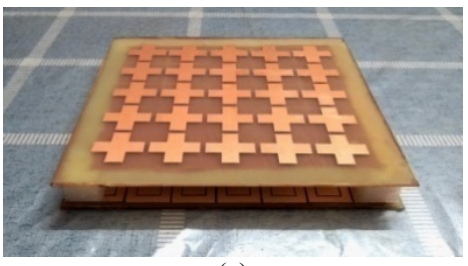

(c)

Fig. 7. FPCA: (a) 3D view, (b) end view and (c) fabricated prototype.
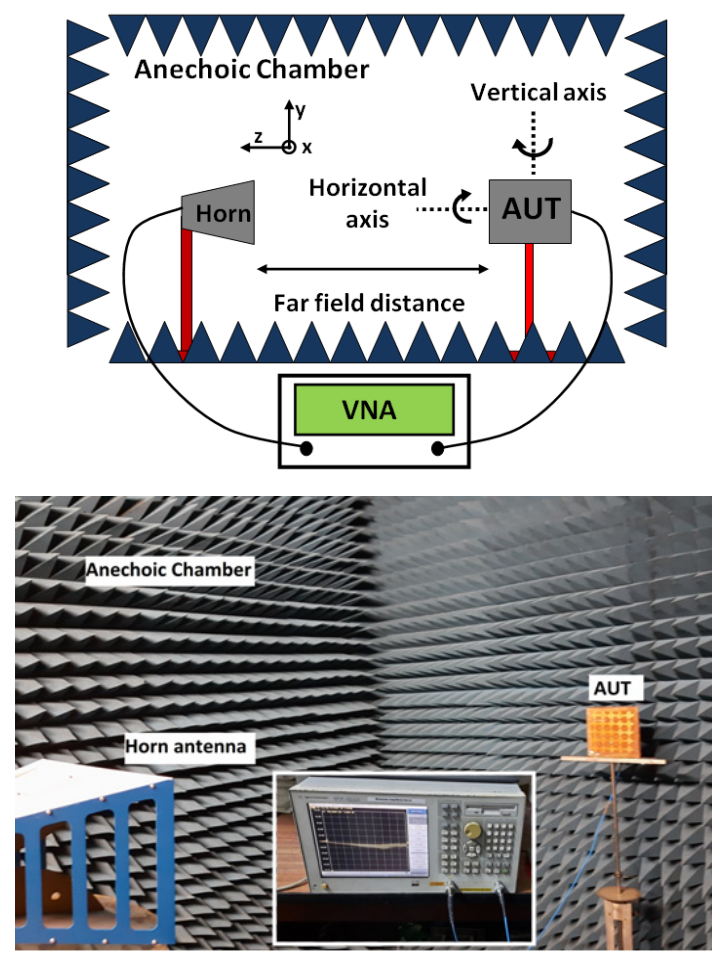

Fig. 8. Illustrated measurement setup of the antenna (top) and actual measurement setup (bottom) with VNA (inset of bottom figure).

The radiation patterns, gains and axial ratio of the AUT are measured inside an anechoic chamber. The measurement setup is shown in Fig. 8.

To measure the gain of the AUT, the gain comparison method is used. In this method, two identical $800 \mathrm{MHz}$ $18 \mathrm{GHz}$ double ridged linearly polarized broadband horn antennas (Model HA-08M18G-NF) whose gains are exactly known (gain standards) are connected to the two ports of the VNA. The antennas are aligned in terms of polarization and direction of maximum radiation intensity. The distance separating the antennas is large enough to ensure that they are in the far-field with respect to one another. The magnitude of $S_{21}$ of the VNA is noted. Next, the horn antenna connected to port 2 is replaced by the AUT. The distance between the antennas is kept the same. The magnitude of $S_{21}$ is noted again. Since the $S_{21}$ of the VNA is related to the ratio of the power received to the power transmitted, Friis' transmission equation can be used to form two sets of equations involving the two measured $S_{21}$ and hence the gain of the AUT can be determined. This method is used to calculate the gain of the antenna at $5.8 \mathrm{GHz}$.

It should be noted however that since the AUT has CP at $2.4 \mathrm{GHz}$, the horn antenna of port 1 has to be oriented once along the horizontal direction to act as a horizontally polarized gain standard and once along the vertical direction to act as a vertically polarized gain standard. Doing this helps us capture the partial gains corresponding to each linearly polarized component of the AUT at $2.4 \mathrm{GHz}$. Then the horizontal and vertical gains can be added to get the total gain of the AUT at $2.4 \mathrm{GHz}$. 
To measure the radiation pattern at $5.8 \mathrm{GHz}$, the standard horn antenna is connected to port 1 of the VNA while the AUT is connected to port 2 such that their principle E-planes lie in the $y z$ plane. The AUT is then rotated about the vertical axis of Fig. 8 (top) from $-150^{\circ}$ to $150^{\circ}$ with an incremental step width of $10^{\circ}$. The magnitude of $\mathrm{S}_{21}$ is noted for each rotation and the Friis' transmission equation is used in each case to map out the radiation pattern in the $x z$ plane. To plot the radiation pattern in the $y z$ plane, both the AUT and the horn antenna are rotated $90^{\circ}$ about the horizontal axis of Fig. 8(top). Now their principle E planes lie in the $x z$ plane. The AUT is again rotated from $-150^{\circ}$ to $+150^{\circ}$ in a similar fashion and the $S_{21}$ are noted to map the radiation pattern in the $y z$ plane.

The radiation pattern at $2.4 \mathrm{GHz}$ is measured similarly but with a slight modification since the antenna contains two orthogonal linearly polarized components. To capture the total radiation pattern in a given plane, the horn antenna has to be oriented vertically once and horizontally once (keeping the orientation of the AUT fixed) and the radiation patterns for these two cases need to be added.

To measure the AR of the AUT at $2.4 \mathrm{GHz}$, the horn antenna is again connected to port 1 while the AUT is connected to port 2 of the VNA. The AUT is then rotated in the $x y$ plane (about the horizontal axis of Fig. 8(top)). The magnitudes and phases of $S_{21}$ are noted for two orthogonal orientations of $\varphi=0^{\circ}$ and $90^{\circ}$. The ratio of these two magnitudes is close to 1 and the phase difference between them is close to $90^{\circ}$. Two additional orthogonal orientations of $\varphi=45^{\circ}$ and $135^{\circ}$ are also chosen and it is seen that even in these orientations, the ratio of the two $S_{21}$ magnitudes is close to 1 and their phase difference is close to $90^{\circ}$.

\subsection{Measured Results}

The reflection coefficient curves of the FPCA are shown in Fig. 9. The red dashed line labeled "Patch" in both Fig. 9(a) and 9(b) represents the $S_{11}$ of the unloaded patch antenna (described in Sec. 3). It can be seen that there is not much difference between the $S_{11}$ of the unloaded patch and the FPCA. This means that the loading effect of the AMC ground plane and the PRS layer on the patch antenna is minimal. The FPCA achieves a $-10 \mathrm{~dB} \mathrm{~S} \mathrm{~S}_{11}$ BW of $8.1 \%(2.31 \mathrm{GHz}-2.52 \mathrm{GHz})$ in the $2.4 \mathrm{GHz}$ band and $6.1 \%(5.61 \mathrm{GHz}-5.96 \mathrm{GHz})$ in the $5.8 \mathrm{GHz}$ band.

It can be observed from Fig. 10 that the loading of the AMC ground and PRS layer slightly increases the AR magnitude of the patch antenna without changing the $3 \mathrm{~dB}$ AR-BW. The FPCA achieves a $3 \mathrm{~dB}$ AR-BW of $7.9 \%$ (2.31 GHz-2.49 GHz).

Near field contour plots shown in Fig. 11 demonstrate the fact that the E field magnitude is uniform throughout the FP cavity at $2.4 \mathrm{GHz}$ while it is concentrated near the central portion of the cavity at $5.8 \mathrm{GHz}$.

The gain enhancements achieved by the FPC at both $2.4 \mathrm{GHz}$ and $5.8 \mathrm{GHz}$ are clearly visible by observing the radiation patterns of Fig. 12. Compared to the unloaded patch

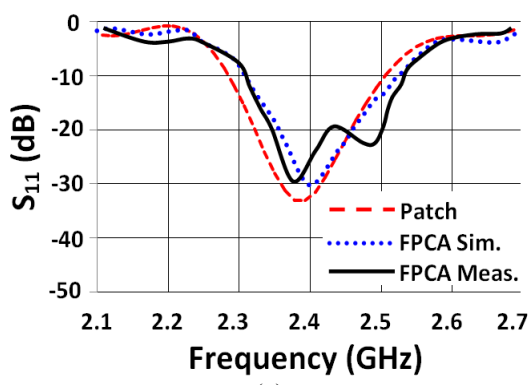

(a)

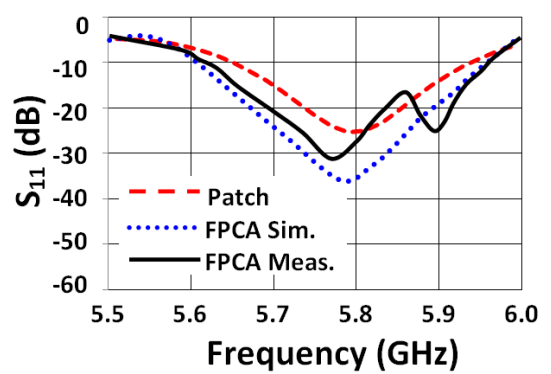

(b)

Fig. 9. $\mathrm{S}_{11}$ vs. frequency plots in the (a) $2.4 \mathrm{GHz}$ band and (b) $5.8 \mathrm{GHz}$ band.

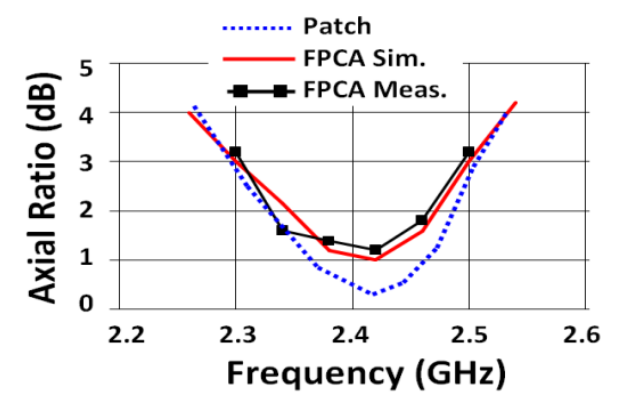

Fig. 10. Axial ratio vs. frequency in the $2.4 \mathrm{GHz}$ band.

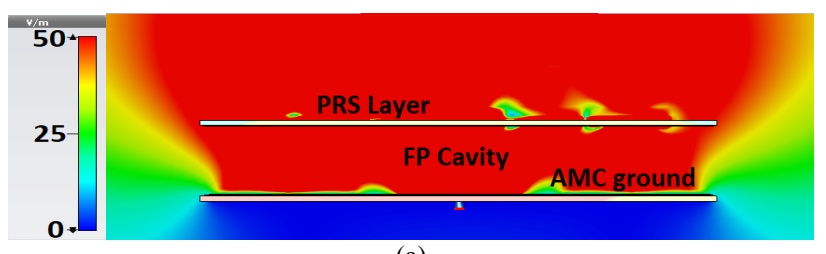

(a)

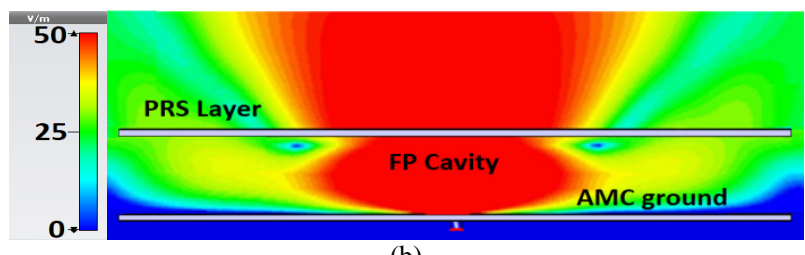

(b)

Fig. 11. E field distributions (V/m) within the FP cavity at (a) $2.4 \mathrm{GHz}$ and (b) $5.8 \mathrm{GHz}$

antenna, the gain of the FPCA is increased by $9 \mathrm{~dB}$ in the first band and $8 \mathrm{~dB}$ in the second band. The proposed antenna has a peak measured gain of $14.3 \mathrm{dBi}(14.1 \mathrm{dBic}$ RHCP; simulated) at $2.4 \mathrm{GHz}$ and $15.5 \mathrm{dBi}$ at $5.8 \mathrm{GHz}$.

The antenna achieves high efficiencies in both the $2.4 \mathrm{GHz}$ band and $5.8 \mathrm{GHz}$ band. The simulated efficiency of the antenna is found to be $\geq 84 \%$ within the $2.4 \mathrm{GHz}$ 
band and $\geq 87 \%$ within the $5.8 \mathrm{GHz}$ band. The radiation efficiency versus frequency plot is shown in Fig. 13.

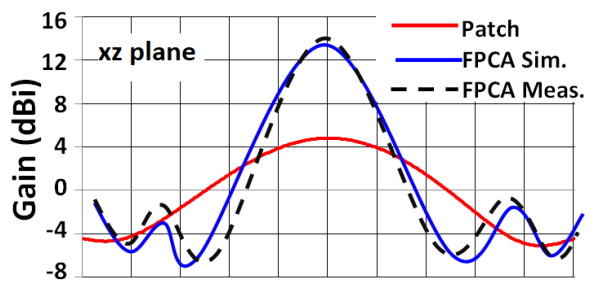

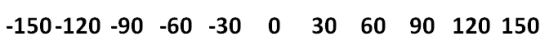
Theta (degrees)

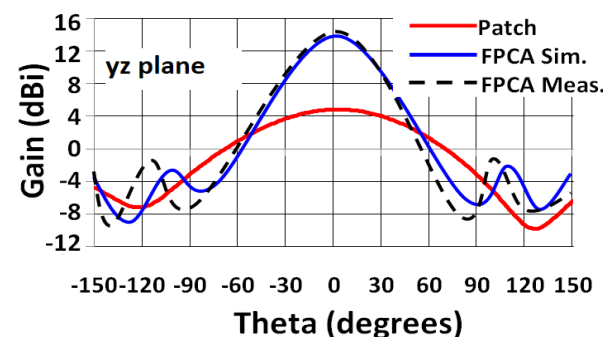

(a)
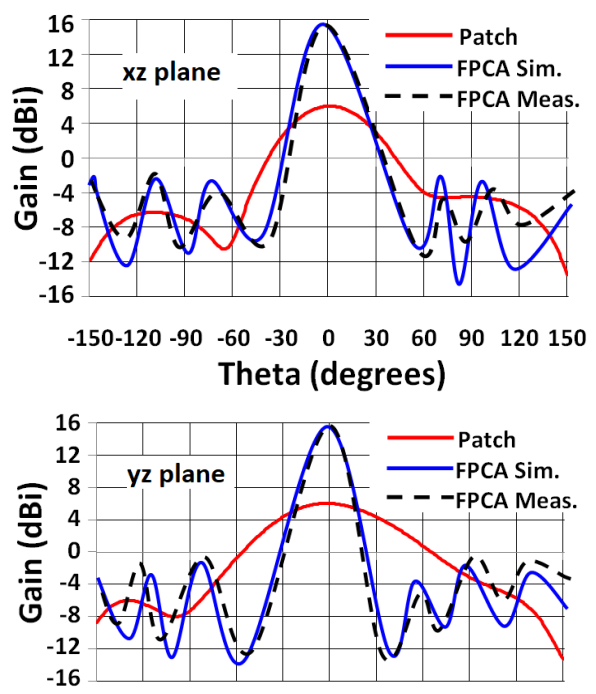

$\begin{array}{llllllllll}-150-120 & -90 & -60 & -30 & 0 & 30 & 60 & 90 & 120 & 150\end{array}$ Theta (degrees)

(b)

Fig. 12. Far field radiation patterns at (a) $2.4 \mathrm{GHz}$ and (b) $5.8 \mathrm{GHz}$.

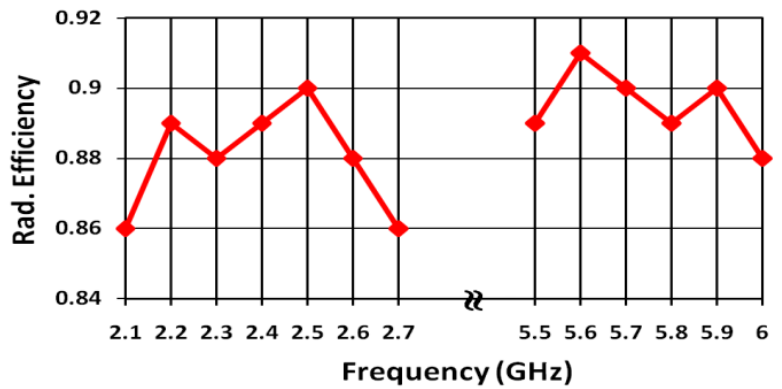

Fig. 13. Simulated radiation efficiencies at the two bands.

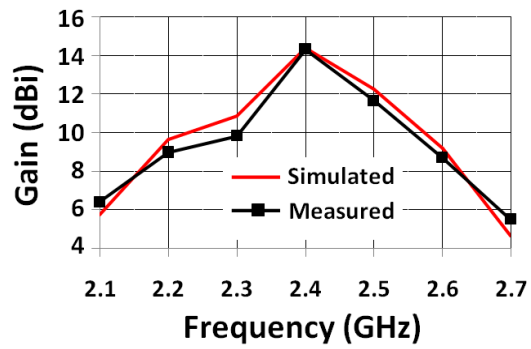

(a)

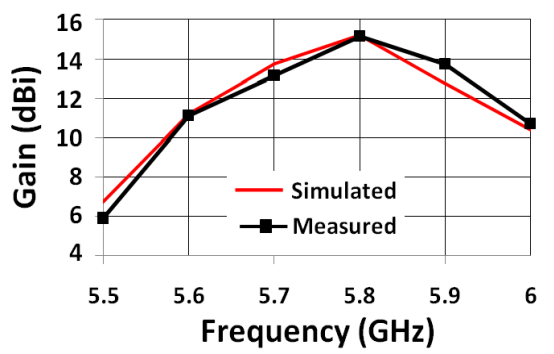

(b)

Fig. 14. Peak gain vs. frequency plots in the (a) $2.4 \mathrm{GHz}$ band and (b) $5.8 \mathrm{GHz}$ band.

Figure 14 presents the peak gain vs. frequency plots of the FPCA in both the frequency bands. The antenna achieves a $3 \mathrm{~dB}$ gain $\mathrm{BW}$ of $7.8 \%(2.34 \mathrm{GHz}-2.53 \mathrm{GHz})$ in the first band and $5.5 \%(5.64 \mathrm{GHz}-5.96 \mathrm{GHz})$ in the second band. Therefore, the proposed antenna achieves very good $3 \mathrm{~dB}$ gain BWs using only a single PRS layer.

In Tab. 1, the proposed antenna is compared with other dual band FPCAs found in literature. The proposed antenna has the smallest cavity height among all the antennas presented. It also has the highest $3 \mathrm{~dB}$ gain $\mathrm{BWs}$ in both the

\begin{tabular}{|c|c|c|c|c|c|c|}
\hline Ref. & No. of PRS layers & $\begin{array}{c}\text { Frequency bands } \\
\text { (GHz) }\end{array}$ & $\begin{array}{c}\text { Cavity height } \\
\text { (w.r.t lower band) }\end{array}$ & $\begin{array}{c}\text { Lateral } \\
\text { dimensions } \\
\text { (w.r.t lower band) }\end{array}$ & $\begin{array}{l}\text { Gain } \\
\text { (dBi) }\end{array}$ & $\begin{array}{c}\text { 3dB gain BW } \\
(\%)\end{array}$ \\
\hline [13] & 2 & $\begin{array}{l}10.6 \\
13.2\end{array}$ & $1 \lambda 10.6 \mathrm{GHz}$ & $5 \lambda \times 5 \lambda_{10.6 \mathrm{GHz}}$ & $\begin{array}{c}14.5 \\
15\end{array}$ & $\begin{array}{l}4.5 \\
4.6\end{array}$ \\
\hline$[14]$ & 2 & $\begin{array}{c}2.45 \\
5.6\end{array}$ & $0.45 \lambda 2.45 \mathrm{GHz}$ & $1.3 \lambda \times 1.3 \lambda_{2.45 \mathrm{GHz}}$ & $\begin{array}{c}14.9 \\
14\end{array}$ & $\begin{array}{c}7 \\
11\end{array}$ \\
\hline$[15]$ & 1 & $\begin{array}{c}6.95 \\
13\end{array}$ & $0.5 \lambda 6.95 \mathrm{GHz}$ & $2.5 \lambda \times 2.5 \lambda_{6.95 \mathrm{GHz}}$ & $\begin{array}{l}16.5 \\
20.9\end{array}$ & $\begin{array}{l}1.44 \\
3.35\end{array}$ \\
\hline$[16]$ & 1 & $\begin{array}{l}19.75 \\
29.75\end{array}$ & $0.5 \lambda 19.75 \mathrm{GHz}$ & $5.3 \lambda \times 5.3 \lambda_{19.75 \mathrm{GHz}}$ & $\begin{array}{l}16.2 \\
18.5\end{array}$ & $\begin{array}{l}4.5 \\
2.4\end{array}$ \\
\hline$[17]$ & 1 & $\begin{array}{l}5.6 \\
10\end{array}$ & $0.28 \lambda 5.6 \mathrm{GHz}$ & $1.5 \lambda \times 1.5 \lambda_{5.6 \mathrm{GHz}}$ & $\begin{array}{l}10.1 \\
15.2\end{array}$ & $\begin{array}{l}4.1 \\
2.7\end{array}$ \\
\hline This paper & 1 & $\begin{array}{l}2.4 \\
5.8\end{array}$ & $0.18 \lambda 2.4 \mathrm{GHz}$ & $1.5 \lambda \times 1.5 \lambda_{2.4 \mathrm{GHz}}$ & $\begin{array}{c}14.3 \\
(14.1 \mathrm{dBic}) \\
15.5\end{array}$ & $\begin{array}{l}7.8 \\
5.5\end{array}$ \\
\hline
\end{tabular}

Tab. 1. Comparison of the proposed FPCA with dual band FPCAs found in literature. 
bands. Only the FPCA presented in [14] has a higher 3dB gain $\mathrm{BW}$ in the second band. However, it uses two PRS layers to achieve this high gain BW. Therefore, the advantages of the proposed antenna over existing dual band FPCAs are clearly highlighted in Tab. 1.

\section{Conclusion}

A dual band FPCA operating at $2.4 \mathrm{GHz}$ and $5.8 \mathrm{GHz}$ is presented. The antenna uses a single PRS layer with metallization on both sides of the substrate to achieve wide $3 \mathrm{~dB}$ gain BWs at both the bands. An AMC ground plane is used to reduce the cavity height of the antenna significantly. The antenna radiates a RHCP wave at $2.4 \mathrm{GHz}$ and a LP wave at $5.8 \mathrm{GHz}$. It achieves high gains and efficiencies in both the $2.4 \mathrm{GHz}$ and $5.8 \mathrm{GHz}$ bands. The proposed antenna is also simple to design and easy to fabricate.

\section{References}

[1] KUKREJA, J., CHOUDHARY, D. K., CHAUDHARY, R. K. A metamaterial inspired ZOR antenna using IDC and spiral inductor with partial ground plane for WLAN applications. Wireless Personal Communications, 2019, vol. 107, p. 137-147. DOI: $10.1007 / \mathrm{s} 11277-019-06244-\mathrm{x}$

[2] KUKREJA, J., CHOUdHARY, D. K., CHAUDHARY, R. K. A short-ended compact metasurface antenna with interdigital capacitor and U-shaped strip. Wireless Personal Communications, 2019, vol. 108, p. 2149-2158. DOI: 10.1007/s11277-019-06514-8

[3] CHOUDHARY, D. K., CHAUDHARY, R. K. Compact filtering antenna using asymmetric CPW-fed based CRLH structure. $A E U$ International Journal of Electronics and Communications, 2020, vol. 126, p. 1-6. DOI: 10.1016/j.aeue.2020.153462

[4] TRENTINI, G. V. Partially reflecting sheet arrays. IRE Transactions on Antennas and Propagation, 1956, vol. AP-4, no. 4, p. 666-671. DOI: 10.1109/TAP.1956.1144455

[5] MU, J., WANG, H., WANG, H., HUANG, Y. Low-RCS and gain enhancement design of a novel partially reflecting and absorbing surface antenna. IEEE Antennas and Wireless Propagation Letters, 2017, vol. 16, p. 1903-1906.DOI: 10.1109/LAWP.2017.2685623

[6] AKBARI, M., GUPTA, S., FARAHANI, M., et al. Gain enhancement of circularly polarized dielectric resonator antenna based on FSS superstrate for MMW applications. IEEE Transactions on Antennas and Propagation, 2016, vol. 64, no. 12, p. 5542-5546. DOI: 10.1109/TAP.2016.2623655
[7] WEILY, A. R., BIRD, T. S., GUO, Y. J. A reconfigurable highgain partially reflecting surface antenna. IEEE Transactions on Antennas and Propagation, 2008, vol. 56, no. 11, p. 3382-3390. DOI: $10.1109 /$ TAP.2008.2005538

[8] ABBOU, D., VUONG, T. P., TOUHAMI, R., et al. High-gain wideband partially reflecting surface antenna for $60 \mathrm{GHz}$ systems. IEEE Antennas and Wireless Propagation Letters, 2017, vol. 16, p. 2704-2707. DOI: 10.1109/LAWP.2017.2742862

[9] RAZI, Z. M., REZAEI, P., VALIZADE, A. A novel design of Fabry-Perot antenna using metamaterial superstrate for gain and bandwidth enhancement. AEU International Journal of Electronics and Communications, 2015, vol. 69, no. 10, p. 1525-1532. DOI: 10.1016/j.aeue.2015.05.012

[10] WANG, N., LIU, Q., WU, C., et al. Wideband Fabry-Perot resonator antenna with two complementary FSS layers. IEEE Transactions on Antennas and Propagation, 2014, vol. 62, no. 5, p. 2463-2471. DOI: 10.1109/TAP.2014.2308533

[11] KONSTANTINIDIS, K., FERESIDIS, A. P., HALL, P. S. Multilayer partially reflective surfaces for broadband Fabry-Perot cavity antennas. IEEE Transactions on Antennas and Propagation, 2014, vol. 62, no. 7, p. 3474-3481. DOI: 10.1109/TAP.2014.2320755

[12] LALBAKHSH, A., AFZAL, M. U., ESSELlE, K. P., et al. Singledielectric wideband partially reflecting surface with variable reflection components for realization of a compact high-gain resonant cavity antenna. IEEE Transactions on Antennas and Propagation, 2019, vol. 67, no. 3, p. 1916-1921. DOI: 10.1109/TAP.2019.2891232

[13] ZEB, B. A., GE, Y.., ESSELLE, K. P., et al. A simple dual-band electromagnetic band gap resonator antenna based on inverted reflection phase gradient. IEEE Transactions on Antennas and Propagation, 2012, vol. 60, no. 10, p. 4522-4529. DOI: 10.1109/TAP.2012.2207331

[14] ABDElghani, M. L., ATTIA, H., DENIDNI, T. A. Dual- and wideband Fabry-Pérot resonator antenna for WLAN applications. IEEE Antennas and Wireless Propagation Letters, 2017, vol. 16, p. 473-476. DOI: 10.1109/LAWP.2016.2585087

[15] MENG, F., SHARMA, S. K. A dual-band high-gain resonant cavity antenna with a single layer superstrate. IEEE Transactions on Antennas and Propagation, 2015, vol. 63, no. 5, p. 2320-2325. DOI: 10.1109/TAP.2015.2405082

[16] LIMA, E. B., COSTA, J. R., FERNANDES, C. A. Multiple-beam focal-plane dual-band Fabry-Pérot cavity antenna with reduced beam degradation. IEEE Transactions on Antennas and Propagation, 2019, vol. 67, no. 7, p. 4348-4356. DOI: 10.1109/TAP.2019.2911365

[17] CHEN, J., ZHAO, Y., GE, Y., et al. Dual-band high-gain FabryPérot cavity antenna with a shared aperture FSS layer. IET Microwaves, Antennas and Propagation, 2018, vol. 12, no. 13, p. 2007-2011. DOI: 10.1049/iet-map.2018.5183 